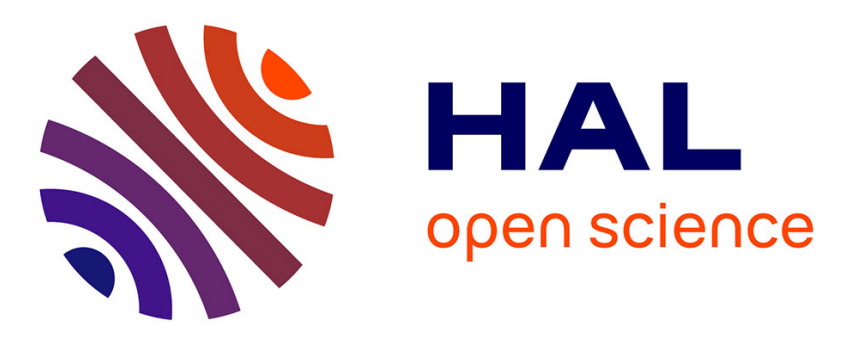

\title{
Characterization of ultrahigh molar mass polyelectrolytes by capillary electrophoresis
}

Laurent Leclercq, Xiaoling Leclercq, Alexis Guillard, Laurent Rodriguez, Olivier Braun, Cédrick Favero, Herve Cottet

\section{- To cite this version:}

Laurent Leclercq, Xiaoling Leclercq, Alexis Guillard, Laurent Rodriguez, Olivier Braun, et al.. Characterization of ultrahigh molar mass polyelectrolytes by capillary electrophoresis. Journal of Chromatography A, 2020, 1631, pp.461536. 10.1016/j.chroma.2020.461536 . hal-03019666

\section{HAL Id: hal-03019666 https://hal.science/hal-03019666}

Submitted on 18 Dec 2020

HAL is a multi-disciplinary open access archive for the deposit and dissemination of scientific research documents, whether they are published or not. The documents may come from teaching and research institutions in France or abroad, or from public or private research centers.
L'archive ouverte pluridisciplinaire HAL, est destinée au dépôt et à la diffusion de documents scientifiques de niveau recherche, publiés ou non, émanant des établissements d'enseignement et de recherche français ou étrangers, des laboratoires publics ou privés. 


\section{Characterization of ultrahigh molar mass polyelectrolytes by capillary electrophoresis}

Xiaoling Leclercq ${ }^{1}$, Laurent Leclercq ${ }^{1}$, Alexis Guillard ${ }^{2}$, Laurent Rodriguez ${ }^{2}$, Olivier Braun $^{2}$, Cédrick Favero ${ }^{2}$ and Hervé Cottet ${ }^{1, *}$

${ }^{1}$ IBMM, University of Montpellier, CNRS, ENSCM, Montpellier, France

${ }^{2}$ SNF Floerger, Andrézieux, France

\section{ABSTRACT}

High to ultrahigh molar mass (above 1 million $\mathrm{g} / \mathrm{mol}$ ) anionic poly(acrylic acid-coacrylamide)s are widely used industrial polymers for water treatment and oil drilling. Their properties are strongly related to their charge density and molar mass distributions. However, due to inherent separation limits of SEC with currently available columns $\left(<5 \times 10^{6} \mathrm{~g} / \mathrm{mol}\right)$ and possible occurrence of chain breakage, and/or adsorption leading to abnormal elution, characterization of unusually high molar masses polyelectrolytes is challenging. In this work, we investigate the use of polymer sieving capillary electrophoresis for the size-based characterization of these high to ultrahigh molar mass polyelectrolytes. By optimizing the operating conditions (electric field, ionic strength, injected polyelectrolyte concentration, nature of the polymer sieving), it has been possible to considerably reduce polyelectrolyte aggregation and to get sufficient size-based selectivity, allowing to obtain the size distribution of the polyelectrolytes over a large range of molar mass from $10^{5}$ up to $\sim 10 \times 10^{6} \mathrm{~g} / \mathrm{mol}$. The data processing of the raw electropherograms is a key step in the analytical protocol leading to the molar mass distribution. The polyelectrolyte effective mobility in sieving conditions has to be normalized to its free-draining electrophoretic mobility in free solution conditions to take into account possible variability in the charge density between the different samples.

Keywords: high molar mass polymer, capillary gel electrophoresis, sieving, molar mass distribution, polyacrylamide derivatives

\section{* CORRESPONDING AUTHOR}

Tel: +33 46714 3427, Fax: +33 46763 1046. E-mail: herve.cottet@ umontpellier.fr 


\section{Introduction}

High to ultrahigh molar mass (> $1 \mathrm{MDa}$ ) anionic polyacrylamides and their analogues are polyelectrolytes which are widely used as flocculent in wastewater treatment [1], as soil conditioner [2], as drag reducer [3], and as viscosity enhancer in Enhanced Oil Recovery for flooding of trapped crude oil from the reservoir [4]. In the oil field application, however, loss of flooding efficiency often arises from shear sensitive chain scissions in porous media and rheological performance deterioration provoked by salinity and high temperature $[5,6]$. It is therefore desirable to thoroughly characterize these polyelectrolytes including their chemical nature (charge density) and molar mass distributions, and to study the impacts of shear stress, salinity and temperature on stability and rheological behaviors for optimal operations. Nevertheless, the characterization of such ultra-high molar mass polyelectrolytes with broad molar mass dispersion using classical methods such as size exclusion chromatography (SEC) is challenging due to the occurrence of chain breakage in size-exclusion chromatography, possible adsorption leading to abnormal elution [7-11], and especially the limited range of the molar mass analyzable with currently available SEC columns. In-batch techniques such as multi-angle laser light scattering (MALLS) [12,13], or open-medium separation techniques such as Field-Flow Fractionation (FFF) [14-17] can be used with a certain success to extend the analysis limits.

Capillary Electrophoresis (CE) is also an attractive separation technique without stationary phase, which was originally mainly developed for biomacromolecules such as DNA [18], proteins [19] and polysaccharides [20]. More recently, $\mathrm{CE}$ has been used for the characterization of synthetic polyelectrolytes [21-26], owing to its rapidity of analysis, minor sample consumption and orthogonal separation mechanism compared to chromatography. Free solution capillary electrophoresis allows to characterize the polyelectrolytes according to their charge density since the polyelectrolyte electrophoretic mobility in absence of gel is independent of the molar mass, due to free draining behavior above a typical molar mass of about $20,000 \mathrm{~g} / \mathrm{mol}$ [27]. It was demonstrated that the distribution in charge density and the associated dispersity indexes can be determined by free solution $\mathrm{CE}[24,25]$. The characterization of highly charged polyelectrolytes above the Manning condensation threshold [28] is challenging, due to the low dependence of the effective mobility against the polyelectrolyte charge density above this threshold [29-31]. Indeed, due to counter-ion condensation, the polyelectrolyte effective charge density levels off above the Manning condensation threshold. Despite the low selectivity, it is however still possible to characterize 
the charge density distribution of highly charged polyelectrolyte by adjusting the ionic strength of the background electrolyte in counter electroosmotic mode free solution CE [32]. Increasing the ionic strength lowers the electroosmotic mobility, and consequently the apparent polyelectrolyte electrophoretic mobility, resulting in a better selectivity of the separation according to the charge density.

For polyelectrolytes of constant charge-to-mass ratio, Capillary Gel Electrophoresis (CGE) using entangled or diluted neutral sieving polymer solution, can be employed for molar massbased separation of polyelectrolytes [18]. The electrophoretic migration of a polyelectrolyte through a polymer network has been elucidated via different separation mechanisms, depending on shape and size of the solute relative to the pore (or blob) size, and on the applied electric field [33, 34]. In the Ogston model [35], a small sized solute is described as a rigid sphere migrating through a porous medium with a relatively large pore size. The reptation model $[36,37]$ depicts a snake-like motion of a solute that is too large to pass freely through the pores of the network and must deform / unfold to pass through the matrix. The biased reptation migration (BRM) [21, 38, 39] describes the forced reptation of a polyelectrolyte under electric field, with orientation and stretching of the chain, leading to electric field- and size-dependent effective electrophoretic mobility, $\mu[21,39]$ :

$$
\mu:\left(\frac{1}{N}+b(E / T)^{2}\right)
$$

where $b$ is a function related to the solute and the network, and $N$ is the number of monomers of the polyelectrolyte. For polymer size comparable to or smaller than the pore size (or blob size) and at low electric field, $\mu$ is a linear function of the inversed size, corresponding to pure reptation regime. For large polyelectrolytes, the size-dependence of the mobility disappears, and the electric field term becomes predominant. These regimes were both identified for DNA separation, as well as for synthetic polyelectrolytes such as polystyrene sulfonates (PSS) [33, $34,40]$.

In this work, we report the size-based characterization of high to ultra-high anionic poly(acrylic acid-co-acrylamide) (molar mass ranging between $22 \mathrm{kDa}-15 \mathrm{MDa}$ ), including standards and industrial samples with charge density comprises between 25 - 33\% (expressed as a molar content in acrylic acid) by CGE using replaceable polymer solution as sieving matrix. A thorough optimization of the operating conditions including the nature of the separating polymer, the electric field strength, the ionic strength and the sample 
concentration, was investigated to permit the molar mass characterization of the APAM samples.

\section{Experimental}

\subsection{Chemicals}

Tris(hydroxymethyl)aminomethane (Tris), anthraquinone-2-sulfonic acid sodium salt monohydrate $97 \%$, hydroxyethylcellulose (HEC, $M_{w} 1.3 \times 10^{6} \mathrm{~g} / \mathrm{mol}$ ) and polyethylene oxide (PEO, average $M_{w} 2 \times 10^{6} \mathrm{~g} / \mathrm{mol}, 5 \times 10^{6} \mathrm{~g} / \mathrm{mol}$ and $8 \times 10^{6} \mathrm{~g} / \mathrm{mol}$ ) were purchased from Aldrich (Saint Quentin Fallavier, France), pullulan from Aureobasidium pullulans $\left(M_{w} 575 \times\right.$ $10^{3} \mathrm{~g} / \mathrm{mol}$ ) from Chem Cruz (Heidelberg, Germany), dextran from Leuconostoc mesenteroids $\left(M_{w} 1.5-2.8 \times 10^{6} \mathrm{~g} / \mathrm{mol}\right)$ from Sigma (Saint Quentin Fallavier, France), dextran SGO $\left(M_{w}\right.$ $50 \times 10^{6} \mathrm{~g} / \mathrm{mol}$ ) kindly provided by SNF (Andrézieux, France), PEO $\left(M_{w} 1 \times 10^{6} \mathrm{~g} / \mathrm{mol}\right)$ from Alfa Aesar (Illkirch, France), hydrochloric acid $37 \%$ and potassium chloride $99.6 \%$ from VWR (Fontenay-sous-Bois, France), sodium chloride 99.5\% from Fluka (Illkirch, France), lithium chloride anhydrous 99\% and lithium hydroxide 98\% from Sigma-Aldrich (Saint Quentin Fallavier, France) and boric acid from Fluka (Illkirch, France).

\subsection{Samples}

All samples of anionic poly(acrylic acid-co-acrylamide) (APAM) in white powder were provided by SNF. Standard APAM samples were synthesized from monomers of sodium acrylate and acrylamide by Controlled Radical Polymerization (CRP) leading to narrow distributed molar mass and anionicity [41]. Industrial samples of APAM (labelled as IPAM) were obtained by hydrolysis of polyacrylamide in a $\mathrm{NaOH}$ medium to reach expected anionicity. The molar mass and PDI were determined by Size Exclusion Chromatography with a Multi Angle Laser Light Scattering detector (SEC-MALS) [11] for the APAM standards, and by batch Static Light Scattering (SLS) for the industrial samples (IPAM). PDI and weight-average molar masses are gathered in Table 1.

For capillary electrophoresis, all samples were used as received and non-filtered. Stock polyelectrolyte solutions were prepared at $5 \mathrm{~g} / \mathrm{L}$ as following: $1 \mathrm{~g}$ sample was carefully added into the vortex formed by a pitched-blade impeller in $200 \mathrm{~mL}$ buffer and agitated at $500 \mathrm{rpm}$ for $6 \mathrm{~h}$. As buffer system $20 \mathrm{mM}$ Tris/Cl buffer containing $35.5 \mathrm{mM} \mathrm{LiCl,} \mathrm{pH} 8.0$ was selected, and as comparison, a borate buffer $(50 \mathrm{mM}$ boric acid /Li buffer containing $7 \mathrm{mM}$ $\mathrm{LiCl}, \mathrm{pH}$ 8.0) was also tested. Before injection, the stock solutions were diluted with the 
background electrolyte (i.e. the sieving medium excluding the sieving polymer) to the desired injected concentration and homogenized on an orbital mixer for 3 min. Stock solutions kept at $5^{\circ} \mathrm{C}$ were warmed up to room temperature before use.

\subsection{Free solution and Capillary Gel Electrophoresis}

Free solution and CGE were performed on a Beckman P/ACE MDQ apparatus (Sciex, Villebon sur Yvette, France). Capillaries were prepared from a fused silica tubing (Composite Metal Services, Photon Lines, France). Unless otherwise stated, the capillary dimensions were $40 \mathrm{~cm}(30 \mathrm{~cm}$ to the detector $) \times 50 \mu \mathrm{m}$ ID. The capillary was first activated by successive flushing (at 20 psi) with $1 \mathrm{M} \mathrm{NaOH}$ for $30 \mathrm{~min}, 0.1 \mathrm{M} \mathrm{NaOH} 10 \mathrm{~min}$, deionized water 10 min, and finally with the background electrolyte for $10 \mathrm{~min}$. Between runs, capillaries were successively flushed at 20 psi with $1 \mathrm{M} \mathrm{NaOH}$ for $15 \mathrm{~min}, 0.1 \mathrm{M} \mathrm{NaOH}$ for $5 \mathrm{~min}$, deionized water for $5 \mathrm{~min}$ and BGE for $10 \mathrm{~min}$. The BGE for free solution CE consisted in a $20 \mathrm{mM}$ Tris/Cl buffer solution of $\mathrm{pH} 8.0$ containing varying $\mathrm{LiCl}$ concentration $(n \mathrm{mM})$ providing a total ionic strength of $(n+11) \mathrm{mM}$. A $100 \mathrm{mM}$ borate/Li buffer $\mathrm{pH} 8.0$ containing varying $\mathrm{LiCl}$ concentration $(n \mathrm{mM})$ was also used, the total ionic strength being $(7+n) \mathrm{mM}$. For CGE, the sieving polymers (pullulan, dextran, PEO) were used as received except HEC that was purified by dialysis at a cutoff of 50,000 against deionized water overnight and then lyophilized. The BGE was made up by dissolving the sieving polymer in the buffer of selected ionic strength under gentle magnetic agitation overnight.

Before injection, $1 \mathrm{~mL}$ of APAM sample solution was obtained by dilution of the stock 5 g/L APAM solution in the BGE (without sieving polymer) to the desired injected concentration (comprised between 0.5 and $2 \mathrm{~g} / \mathrm{L}$ ), mixed with $25 \mu \mathrm{L}$ of DMF (at $0.2 \%$ in water, electro-osmotic flow marker) and $20 \mu \mathrm{L}$ of anthraquinone-2-sulfonic acid sodium salt ( $2 \%$ in water, mobility marker) on an orbital mixer. Samples were injected at 0.5 psi for $5 \mathrm{~s}$. Separation voltage of $+2 \mathrm{kV}$ (unless otherwise stated) was applied in normal mode (i.e. positive polarity with counter-electroosmotic mode). UV absorption was monitored at 200 $\mathrm{nm}$. Temperature of the sample chamber and of the capillary cassette was set at $25^{\circ} \mathrm{C}$.

\subsection{Data processing of the electropherograms}

The electropherograms were transformed into effective mobility scale. The effective mobility was calculated as: 


$$
\mu=\left(\frac{1}{t}-\frac{1}{t_{\text {eof }}}\right) \frac{l L}{V}
$$

166

167

168

169

170

171

172

173

where $t_{\text {eof }}$ is the elution time of the neutral marker (DMF), $t$ the migration time of the solute, $l$ the effective capillary length to the detector, $L$ the total capillary length, $V$ the separation electric voltage. In this transformation, the y-axis was transformed to a mobility massweighted distribution function $\mathrm{P}(\mu)$ using the following equation:

$$
P(\mu)=\left(\frac{A(t) / t}{|d \mu / d t|}\right) \approx\left(\frac{A(t) / t}{1 / t^{2}}\right)=A(t) \times t
$$

with $A(t)$ the UV absorbance of the detector [24]. The average effective mobility of the polymer calculated on the whole polymer distribution was determined according to:

$$
\bar{\mu}=\frac{\int P(\mu) \mu d \mu}{\int P(\mu) d \mu}=\frac{\int A(t) t \mu d \mu}{\int A(t) t d \mu}
$$

The detector signal is recorded in digitalized form of $N_{\text {data }}$ data points $\left(A\left(t_{i}\right) ; t_{i}\right)$ varying from the initial $(i=1)$ to the end time point ( $i=N_{\text {data }}$ ) of the polymer signal, eq (4a) can be rewritten in its discretized form $(4 b)$ :

$$
\bar{\mu}=\frac{\sum_{i=1}^{N_{\text {data }}} A\left(t_{i}\right) t_{i} \mu_{i}\left(\mu_{i+1}-\mu_{i}\right)}{\sum_{i=1}^{N_{\text {data }}} A\left(t_{i}\right) t_{i}\left(\mu_{i+1}-\mu_{i}\right)}
$$

To get the molar mass distribution, it is first required to normalize the effective mobility $\mu$ obtained in sieving conditions to the average free-solution effective mobility $\bar{\mu}_{0}$ obtained in the same electrophoretic conditions but in the absence of sieving matrix. This normalization is important to take into account to correct from the possible differences in polyelectrolyte charge density from one sample to the other. For that, the effective mobility distributions were transformed in distribution of $\mu / \bar{\mu}_{0}$ using the following equation:

$$
P\left(\frac{\mu}{\bar{\mu}_{0}}\right)=\frac{P(\mu)}{\frac{d\left(\frac{\mu}{\bar{\mu}_{0}}\right)}{d \mu}} \approx A(t) \times t \times \bar{\mu}_{0}
$$


Assuming the charge density of samples was evenly distributed according to the molar mass distribution, the molar mass distribution $P\left(M_{w}\right)$ is obtained using a calibration curve obtained with APAM standards according to equations (5) and (6) [24]:

$$
\frac{\mu}{\bar{\mu}_{0}}=\alpha M_{w}^{\beta}
$$

$$
P(M)=\frac{P\left(\frac{\mu}{\bar{\mu}_{0}}\right)}{\left|\frac{d M}{d \frac{\mu}{\bar{\mu}_{0}}}\right|}=\alpha^{1 / \beta} \beta\left(\frac{\mu}{\bar{\mu}_{0}}\right)^{\frac{\beta-1}{\beta}} \times A(t) \times t \times \bar{\mu}_{0}
$$

The calculation of the weight- and the number-average molar mass leading to the dispersity index can finally be calculated for each sample using equations (8-10):

$$
\begin{aligned}
& M_{w}=\frac{\int P(M) M d M}{\int P(M) d M} \\
& M_{n}=\frac{\int P(M) d M}{\int \frac{P(M)}{M} d M} \\
& P D I=\frac{M_{w}}{M_{n}}
\end{aligned}
$$

\subsection{Size-Exclusion Chromatography (SEC)}

Weight and number average molecular mass, PDI, and radius-of-gyration of APAM standards were collected by a SEC-MALS system. The SEC system used consisted of an on-line degasser, a high-pressure pump (Agilent 1260 Infinity I), an automatic sampler (Agilent 1260 Infinity I) a $8 \times 300 \mathrm{~mm}$ SEC column packed with polyhydroxymethacrylate-based gel (OHpak columns, Shodex, Japan), a MALS detector (Dawn Heleos II, Wyatt Technologies) and a refractive index detector (Optilab, Wyatt Technologies). Experimental conditions of the analysis have been realized according to Jouenne et al. study [11]. A $0.5 M$ sodium nitrate solution and $55 \mathrm{mM}$ HEPES sodium buffer $(\mathrm{pH} \mathrm{8)}$ was used as the eluent. The eluent was filtered through a $0.1 \mu \mathrm{m}$ cellulose membrane before use. The flow rate was set at $0.3 \mathrm{~mL} / \mathrm{min}$ 
$\mathrm{mL} / \mathrm{min}$. Sample concentration of $0.02 \%(\mathrm{w} / \mathrm{V})$ in the eluent. Before injection, the sample solutions were filtered through a $1.2 \mu \mathrm{m}$ cellulose disposable membrane. The injection volume was $100 \mu \mathrm{L}$. The detector cells of MALS and RI were kept at ambient temperature. The value of the refractive index increment $(\mathrm{d} n / \mathrm{d} c)$ of each sample was measured by using the Optilab detector (Wyatt Technology). Data acquisition and processing were carried out using the ASTRA software (version 6.1, Wyatt Technologies).

\subsection{Batch Static Light Scattering (SLS)}

SLS batch analysis was carried out on a Dawn HELEOS II equipment (Wyatt Technology, Toulouse, France). Polymer solutions were prepared in an aqueous mobile phase (0.5 M $\mathrm{NaNO}_{3}$ ) at $0.5 \mathrm{wt} \%$ concentration of polymer and mixed with a mechanical stirrer (at 400 $\mathrm{rpm}$ ), at room temperature for $2 \mathrm{~h}$, to obtain dissolution. The $0.5 \mathrm{wt} \%$ polymer solutions were further diluted to $0.01 \mathrm{wt} \%$ and stirred with a stir bar at $200 \mathrm{rpm}$ for $1 \mathrm{~h}$. The diluted solutions were then filtered through $1.2 \mu \mathrm{m}$ syringe filters to remove dust and any other large particle contaminants. The filtered $100 \mathrm{ppm}$ solutions were diluted sequentially to 4-5 different concentrations with a set of two syringe pumps, and then directly injected into a the MALS detector. The MALS cell temperature was set at $30^{\circ} \mathrm{C}$. The flow rate was $0.3 \mathrm{~mL} / \mathrm{min}$. The scattering data were collected at 17 different angles with an incident laser wavelength of $664 \mathrm{~nm}$. Calibration of the MALS detectors was done using HPLC grade toluene. The data analysis was conducted with Astra 6 software provided by Wyatt Technology (Toulouse, France). In a batch MALS measurement, the angular and concentration dependent light scattering data are fitted with Ornstein-Zernick formalism:

$$
\frac{R}{K C}=M_{w}\left(1+\frac{2 q^{2} R_{g}^{2}}{3 D_{f}}\right)^{\frac{D_{f}}{2}}
$$

where $R$ is the excess Rayleigh ratio, $K$ the optical constant, $C$ the polymer concentration, $M_{w}$ the weight-average molar mass, $D_{f}$ the fractional size, $q$ the scattering factor and $R_{g}$ the radius of gyration.

\section{Results and discussion}

\subsection{Free solution capillary electrophoresis}


The electropherograms of the APAM standards in free solution CE obtained in a $20 \mathrm{mM}$ Tris- $\mathrm{HCl}$ buffer containing $85.5 \mathrm{mM} \mathrm{LiCl}, \mathrm{pH} 8$ (96.5 mM ionic strength) are presented in Fig. 1, both in time (Fig. 1A) and in effective electrophoretic mobility scales (Fig. 1B). LiCl was used instead of $\mathrm{NaCl}$ to reduce the conductivity, since $\mathrm{Li}^{+}$has a lower ionic mobility than $\mathrm{Na}^{+}$. The separation is performed under counter-electroosmotic mode on fused silica capillary, using positive polarity. The transformation of the electropherograms in effective electrophoretic mobility scale allows to get rid of the EOF fluctuations, as it can be observed by the perfect alignment of the acrylic acid peak (peak 3) in Fig. 1B. As observed on the distributions, the effective mobilities of the APAM standards vary between -20 TU and -25 TU (where TU stands for Tiselius Unit, $10^{-9} \mathrm{~m}^{2} \mathrm{~s}^{-1} \mathrm{~V}^{-1}$ ), depending on their polymer charge density distributions. The mobility distributions of the APAM standards largely overlap one each other, with slightly faster average mobility for APAM50 and APAM10, in good agreement with their higher anionicity (see Table 1). The average free solution electrophoretic effective mobility $\bar{\mu}_{0}$ were calculated for each APAM sample according to equation (4b) and are gathered in Table 1.

\subsection{Evaluation of the nature of the sieving polymer}

As a sieving media, several neutral linear polysaccharides or synthetic polymers were considered: pullulan (575 KDa), dextran (1.6-2.8 MDa), dextran SGO (50 MDa), hydroxyethyl cellulose (1.3 MDa) and four polyethylene oxides (PEO) of molar mass varying between 1.5-8 MDa. Polymers with relatively high molar masses were used in order to reach large mesh size or blob size $\xi_{b}$ (i.e. low polymer concentration) in entangled conditions $(C>$ $C^{*}$ ). The entanglement threshold $C^{*}$ depends on the polymer molar mass, and can be derived from the intrinsic viscosity using equation (12) [33]:

$$
C^{*}=\frac{1.5}{[\eta]}
$$

The $C^{*}$ values of the polymers used in this work are given in Table 2 . The 'blob size' $\xi_{b}$ is a decreasing function of the polymer concentration, and does not depend on the polymer molar mass (as far as the solution is entangled, otherwise it is not defined) according to equation (13) [40]: 


$$
\xi_{b}=\left(\frac{4 \pi N_{a}}{3}\right)^{-\frac{1+a}{3 a}}=\left(\frac{K}{6^{3 / 2} \Phi}\right)^{-\frac{1}{3 a}} C^{-\frac{1+a}{3 a}}
$$

where $a$ and $K$ are the Mark-Houwink coefficients, $N_{a}$ the Avogadro constant and $\Phi$ the modified Flory viscosity constant. The characteristics of the polymers including the molar mass $M_{w}$, the entangled threshold $C^{*}$, the $[\eta]-M_{w}$ relationship, the used concentrations $C$, and the blob sizes $\xi_{b}$ at the used concentrations are gathered in Table 2. For HEC, PEO and pullulan, the used concentrations were chosen close to $C^{*}$ to get the largest blob size (164-174 $\mathrm{nm}$ for HEC and PEO) and to limit the viscosity of the entangled polymer solution.

The separation of the 6 APAM standards were investigated using pullulan, dextran, dextran SGO or HEC at $46.5 \mathrm{mM}$ ionic strength and at a constant electric voltage of $+2 \mathrm{kV}(50 \mathrm{~V} / \mathrm{cm})$ (except $+8 \mathrm{kV}$, i.e. $200 \mathrm{~V} / \mathrm{cm}$, for pullulan). The best results were obtained with HEC entangled solution, where the 4 first APAM standards with molar mass between $0.22 \times 10^{6}$ and $3.93 \times 10^{6} \mathrm{~g} / \mathrm{mol}$ were partially separated (see Fig. 2). However, the analysis of APAM50 $4.18 \times 10^{6} \mathrm{~g} / \mathrm{mol}$ and APAM60 $7.34 \times 10^{6} \mathrm{~g} / \mathrm{mol}$ was perturbed by the aggregation phenomenon during the CGE (discussed in the next section), that generates spikes during the electrophoretic separation. Compared to the decreasing mobility with molar mass observed in the presence of HEC, lower (or even zero) selectivity according to the APAM molar mass was recorded in pullulan, dextran or dextran SGO solutions (see Fig. SI1 to SI3), whatever the concentration of the separating polymer tested (see Table 2). The lacking sieving ability of these polymers might be related to a too low molar mass of the polymer for pullulan or by the presence of ramifications for dextrans, which may lead to short mesh lifetime [40]. It can also be explained by differences in chain stiffness (pullulan and dextran, $0.4 \mathrm{~nm}$ persistence length, being much more flexible than rigid HEC 26-40 nm [42]). As for PEO, un-entangled $1.5 \mathrm{~g} / \mathrm{L}$ solutions (PEO 1.5, 2 or $5 \mathrm{MDa}$ ) at $96.5 \mathrm{mM}$ ionic strength provided good molar mass selectivity up to about $3-4 \times 10^{6} \mathrm{~g} / \mathrm{mol}$ (i.e. up to APAM30, see Fig. SI4 A and B), however, the detection sensitivity was overall lower than in the HEC media. The least sensitivity of detection was observed in entangled PEO 8 MDa solution, together with lower baseline stability and aggregation of APAM60 (see Fig. SI4 C and D).

\subsection{Reduction of aggregation phenomenon}

The phenomenon of electrohydrodynamically-induced aggregation was already encountered in CE of long-chain DNA (> $30 \mathrm{kbp}$ ) [43, 44] or polysaccharides [45]. It was 
explained as the electroneutrality breakdown between the polyanions and the co- and counterions of the BGE under action of electric field, that extended on a scale of a few microns far beyond the Debye layer (typically, 1 to $2 \mathrm{~nm}$ ) [43]. The resulted non-linear electric force is capable to produce an electrohydrodynamic flow and in turn concentration fluctuation that leads to the aggregation of the polyanions. The electric field threshold $E_{a}$ at which aggregation occurs is a function of the polymer concentration $C_{p}$ and the electrolyte concentration $C_{s}$ according to the following scaling law:

$$
E_{a} \sim \sqrt{\frac{C_{s} \eta}{C_{p}}}
$$

where $\eta$ is the solution viscosity. Equation (14) predicts that aggregation occurs at higher electric field strength by increasing the electrolyte concentration and/or by decreasing the polymer concentration.

\subsubsection{Impact of ionic strength and sample concentration on aggregation reduction}

In addition to its beneficial effect on aggregation phenomenon, it is known that higher ionic strength decreases the electrophoretic mobility of solutes and electroosmotic mobility [46], and also leads to more compact conformations of polyelectrolytes [47]. The impact of ionic strength on aggregation was significant: by increasing ionic strength from $46.5 \mathrm{mM}$ to $96.5 \mathrm{mM}$ at $2 \mathrm{kV}(50 \mathrm{~V} / \mathrm{cm})$ in the HEC solution, APAM50 and APAM60 aggregated much less, as demonstrated in Fig. 3 with better peak shape for the APAM signals. Further increase of the background electrolyte ionic strength from $96.5 \mathrm{mM}$ to $136.5 \mathrm{mM}$ (or even $196.5 \mathrm{mM}$ ) did not lead to improvement of the separation, while the total run time was severely increased due to decreased apparent electrophoretic mobility, both in sieving and free solution conditions. Thus, $96.5 \mathrm{mM}$ ionic strength was considered as a good balance between separation performance and analysis time. As expected [43], lower injected concentration (1.5 $\mathrm{g} / \mathrm{L}$ vs $2 \mathrm{~g} / \mathrm{L}$ ) also tends to reduce the adverse effect of aggregation. However, due to limited sensitivity of detection of APAM, it was hardly possible to reduce the injected concentration below $0.5 \mathrm{~g} / \mathrm{L}$.

\subsubsection{Impact of the electric field strength on aggregation reduction}

Since aggregation was induced by action of the electric field, decreasing the electric field strength was an effective counteraction. In the HEC solution at the optimized ionic strength of $96.5 \mathrm{mM}$, decreasing the applied separation voltage from $+2 \mathrm{kV}(50 \mathrm{~V} / \mathrm{cm})$ to $+1 \mathrm{kV}(25$ 
$\mathrm{V} / \mathrm{cm}$ ) effectively suppressed the aggregation of high polyelectrolytes (see Fig. SI5), but the peak broadening due to late migration times generated low signal to noise ratio. Therefore, the separation voltage was retained at $+2 \mathrm{kV}$ in the following sections.

\subsection{Effect of the nature of the buffering species on the size-based separation}

Surprisingly, changing the Tris/Li/Cl buffer by a Li/borate/Cl buffer, and keeping constant the other conditions (ionic strength at $\sim 100 \mathrm{mM}, \mathrm{pH} 8$, and $0.8 \mathrm{~g} / \mathrm{L} \mathrm{HEC}$ ), led to much lower size-based selectivity of the APMA standards (see Fig. SI6), indicating Tris/Li/Cl buffer was better suited for HEC sizing of APAMs. One possible explanation of this effect could be that the selectivity obtained in HEC/Tris sieving medium is partly due to a transient interaction between the APAM polyelectrolyte and the neutral separating HEC chain (as already observed for DNA separation [48]), and not only due to a sieving mechanism. In the context of such prevailing separation mechanism, the nature of the buffering ions could change the strength of the APAM / HEC interaction, which would be higher in the case of Tris/Li/Cl buffer compared to $\mathrm{Li} /$ borate/Cl buffer.

\subsection{Reduced electrophoretic mobility distributions}

The effective mobility distributions presented in Fig. 3D were transformed in distribution of $\mu / \bar{\mu}_{0}$ according to equation (5), as presented in Fig. 4A. This transformation aimed at a correction of the impact of slightly higher charge density values for samples APAM 50 and APAM 10, leading to a smoother 'non-biased' variation of the reduced effective mobility according to the molar mass.

Under the optimized conditions established with APAM standards, CGE was performed on 4 industrial samples (IPAM) of molar mass up to $15 \mathrm{MDa}$ (as estimated by SLS, see Table 1) and an anionicity between 27 and 33\%. The results were presented in Fig. 4B. Electropherograms obtained in free solution for the IPAM samples under similar electrophoretic conditions can be found in Fig. SI7.

\subsection{Electrophoretic mobility - molar mass calibration curve}

The size-based selectivity obtained for the APAM (standards) in $0.8 \mathrm{~g} / \mathrm{L} \mathrm{HEC} \mathrm{at}+2 \mathrm{kV}$ and $96.5 \mathrm{mM}$ ionic strength is quantitatively represented on a Ferguson-like plot (see the red dots in Fig. 5), where the $\log$ of the average reduced electrophoretic mobility $\left(\bar{\mu} / \bar{\mu}_{0}\right)$ is 
plotted against $\log M_{w}$. The weight-average molar mass $M_{w}$ of the APAM standards was obtained by SEC-MALS and varied between $0.22 \times 10^{6}$ and $7.34 \times 10^{6} \mathrm{~g} / \mathrm{mol}$ (chromatograms are presented in Figure SI8). The average effective mobility of the polyelectrolyte migrating in the presence of HEC solution $\bar{\mu}$, was calculated according to equation (4) to the average effective mobility in free solution $\left(\bar{\mu}_{0}\right)$. The latter was separately determined in the absence of the sieving solution under the same experimental conditions using equation (4b). A linear log$\log$ correlation calculated on the APAM standards (red dots) was obtained leading to the following scaling law by least-square regression on the APAM standards: $\log \left(\bar{\mu} / \bar{\mu}_{0}\right)=(-$ $0.113 \pm 0.007) \times \log M_{w}-(0.109 \pm 0.004) \quad\left(M_{w}\right.$ in $\left.10^{6} \mathrm{~g} / \mathrm{mol}\right), \mathrm{R}^{2}=0.9864$ or $\bar{\mu} / \bar{\mu}_{0}=$ $(0.778 \pm 0.007) \times M_{w}{ }^{(-0.113 \pm 0.007)}$. As expected, the slope was far from -1 value predicted for a pure reptation regime, suggesting that the migration was strongly electric field-dependent (biased reptation with orientation), and possibly mixed with a transient polyelectrolyte / HEC interaction, as discussed in section 3.4.

\subsection{Calculation of molar mass distribution and polydispersity of industrial samples}

The molar mass distributions $\mathrm{P}(M)$ of the standard and industrial samples were calculated from the electropherogram in the reduced electrophoretic mobility scale according to equation (6) and (7), where the calibration equation is derived from Fig. 5 as $\bar{\mu} / \bar{\mu}_{0}=$ $(0.778 \pm 0.007) \times M_{w}{ }^{(-0.113 \pm 0.007)}$. Fig. 6 displays the area-normalized molar mass distributions $P(\log M) \sim P(M) \times M$ as a function of $\log M$ for the standard and industrial samples. In these optimized conditions, the molar mass distribution can be obtained up to $10 \mathrm{MDa}$ without significant effect of the aggregation. Above $10 \mathrm{MDa}$, the molar mass distribution can be impacted by the aggregation effect as visualized by the multiple spikes for the IPAM 3 and 4 samples (see Fig. 6B). A comparison on the Ferguson-like plot using $M_{w}$ from SLS is displayed in Fig. 5 for the IPAM samples, showing a reasonable extrapolation of the $\log (\bar{\mu} /$ $\left.\bar{\mu}_{0}\right) v s \log M_{w}$ correlation for molar mass higher than the largest APAM standard (i.e. $M_{w}>$ 7.34 MDa). The average molar mass $M_{w}$ (CGE), of the industrial samples calculated by integration of the molar mass distributions according to equation (8), as well as the polydispersity of all samples using equation (10) are gathered in Table 1. The $M_{w}$ values obtained by CGE for most the IPAM industrial samples: 6.5 MDa (IPAM2), 10.1 MDa (IPAM3) and 14.9 MDa (IPAM4), are similar to those obtained by SLS (8, 10.5 and 15 MDa, respectively) within the confidence interval (calculated as \pm one $\mathrm{SD}$ on $n=3$ repetitions). The 
$M_{w}$ CGE values of IPAM1 industrial sample $(4.3 \mathrm{MDa})$ is in better agreement with the SEC value (4.3 MDa), but lower than the SLS one (6.5 MDa).

As for the polydispersity, and as expected, APAM standards have relatively low polydispersity indexes ranging between 1.2 and 1.5 in CGE; which are in reasonably good agreement with those obtained by SEC for most of the APAM samples. Significantly smaller PDI were obtained by CGE for APAM 10 and APAM 50 samples compared to SEC, which may be related to higher peak broadening in SEC compared to CGE, as observed in previous studies $[25,49,50]$. As for IPAM industrial samples, polydispersity indexes are obviously much higher than for APAM samples, and are comprised between 2.4 and 2.9 for IPAM 1 and IPAM 2 samples. It is worth noting that IPAM samples cannot be analyzed by SEC because of shear degradation occurring through the columns, adsorption phenomena and lack of separation due to actual SEC column technology. The main limitation is due to the exclusion limit of the gel matrix used in SEC [11]. IPAM 3 and IPAM 4 polydispersities could not be accurately determined by CGE due to the presence of spikes above $10 \mathrm{MDa}$ (aggregation phenomenon).

On the whole, one can conclude that the $M_{w}$ values obtained by CGE are quite consistent with those obtained from other methods within the experimental confidence intervals. CGE can be used to determine average molar mass, molar mass distribution and polydispersity index of APAM/IPAM samples up to about $10 \mathrm{MDa}$.

\section{Conclusion}

The molar mass-based characterization of anionic polyacrylamides by CGE using entangled HEC solutions as sieving media was successfully achieved up to about 10 MDa by unifying two prerequisites: analysis of high polymers was made possible by aggregation reduction and HEC was capable to size the whole range of the molar masses studied. The sample aggregation was reduced by optimization of the operating parameters: low electric field strength $(50 \mathrm{~V} / \mathrm{cm})$, relatively high ionic strength of $96.5 \mathrm{mM}$, low sample concentration ( $\sim \mathrm{g} / \mathrm{L})$. The buffer nature (TRIS) was also found crucial to get appropriate selectivity that is both depending on sieving mechanism and possible polyelectrolyte / HEC transient interaction. Aggregation was also promoted by the presence of separating polymer, while no detectable aggregation was observed in free solution under the same conditions.

Based on the $\log \left(\bar{\mu} / \bar{\mu}_{0}\right)-\log M_{w}$ relationship established with the APAM standards, the molar mass distribution and the polydispersity index of the APAM and IPAM samples were 
obtained as far as the molar mass did not exceed $10 \mathrm{MDa}$. The molar mass values were similar to those determined by SLS, proving the potential of CGE in molar mass based characterization of high ultra-high molar mass polyelectrolytes up to about $10 \mathrm{MDa}$. Further or future developments may focus on the limitation of the aggregation above this threshold.

\section{Acknowledgments}

This work was partly funded by SNF, under a Cooperative Research and Development Agreement with the University of Montpellier and the CNRS.

\section{References}

[1] A.G. Guezennec, C. Michel, K. Bru, S. Touze, N. Desroche, I. Mnif, M. Motelica-Heino, Transfer and degradation of polyacrylamide-based flocculants in hydrosystems: a review, Environ. Sci. Pollut. Res.

[2] C. Seybold, Polyacrylamide review: soil conditioning and environmental fate, Commun. Soil Sci. Plant Anal. 25 (1994) 2171-2185, doi:10.1080/00103629409369180.

[3] G. Rodvelt, S. Yuyi, C. Van Gilder, Use of a salt-tolerant friction reducer improves production in Utica completions, In SPE Eastern Regional Meeting. Society of Petroleum Engineers, Richardson, TX, USA, 2015.

[4] A. Thomas, N. Gaillard, C. Favero, Some key features to consider when studying acrylamide-based polymers for chemical enhanced oil recovery, Oil Gas Sci. Technol. 67 (2013) 887-902, doi:10.2516/ogst/2012065.

[5] A. Sabhapondit, A. Borthakur, I. Haque, Characterization of acrylamide polymers for enhanced oil recovery, J. Appl. Polym. Sci. 87 (2003) 1869-1878, doi:10.1002/app.11491.

[6] L. Tolstikh, N. Akimov, I. Golubeva, I. Shvetsov, Degradation and stabilization of polyacrylamide in polymer flooding conditions, Int. J. Polym. Mater. 17 (1992) 177-193, doi:10.1080/00914039208041113.

[7] T.Q. Nguyen, H.H. Kausch, Chain scission in transient extensional flow kinetics and molecular weight dependence, J. Non-Newtonian Fluid. Mech., 30 (1988) 125-140, doi:10.1016/0377-0257(88)85020-1.

[8] A. Zaitoun, P. Makakou, N. Blin, R. Al-Maamari, A.A. Al Hashmi, M. Abdel-Goad, H. Al-Sharji, Shear stability of EOR polymers, SPE Journal 17 (2012) 335-339, doi:10.2118/141113-PA.

[9] L. Rodriquez. PhD Thesis : Analyse dimensionnelle, comportement thermique et mécanique de polymères en solution aqueuse à base de 2-acrylamido-2-méthylpropane sulfonate : Application en récupération assistée d'hydrocarbures. Université de Pau et des Pays de l'Adour, France, 2016.

[10] L. Rodriguez, A. Mejia, S. Reynaud, G. Lespes, C. Favero, S. Antignard, B. Giovannetti, N. Gaillard, G. Dupuis, M. Loriau, S. Jouenne, B. Grassl. Monitoring thermal and mechanical stability of enhanced oil recovery (EOR) acrylamide based polymers (PAM) through intrinsic viscosity (IV): Determination using a new capillary rheology technique, SPE EOR Conference at Oil and Gas, West Asia, Muscat, Oman, 21-23 March 2016.

[11] S. Jouenne, M. Loriau, B. Grassl, N. Andreu. Method for determining the weight-average molecular weight of a water-soluble high molecular weight polymer, Patent WO 2017042603 A1 2017031, 2017.

[12] J. Wang, H. Huang, X. Huang, Molecular weight and the Mark-Houwink relation for ultra-high molecular weight charged polyacrylamide determined using automatic batch mode multi-angle light scattering, J. Appl. Polym. Sci. 133 (2016) 43748, doi:10.1002/app.43748.

[13] B.A. Buchholz, A.E. Barron, The use of light scattering for precise characterization of polymers for DNA sequencing by capillary electrophoresis, Electrophoresis 22 (2001) 4118-4128, doi:10.1002/15222683(200111)22:19<4118::AID-ELPS4118>3.0.CO;2-Q.

[14] S. Woo, J. Y. Lee, W. Choi, M. H. Moon, Characterization of ultrahigh-molecular weight cationic polyacrylamide using frit-inlet asymmetrical flow field-flow fractionation and multi-angle light scattering, J. Chromatogr. A 1429 (2016) 304-310, doi:10.1016/j.chroma.2015.12.027. 
[15] Y. Dalsania, A. Doda, J. Trivedi, Characterization of ultrahigh-molecular-weight oilfield polyacrylamides under different $\mathrm{pH}$ environments by use of asymmetrical-flow field-flow fractionation and multiangle-light-scattering detector, SPE Journal 23 (2018) 48-65, doi:10.2118/174624-PA.

[16] M. Leeman, M. T. Islam, W. G. Haseltine, Asymmetrical flow field-flow fractionation coupled with multi-angle light scattering and refractive index detections for characterization of ultra-high molar mass poly(acrylamide) flocculants, J. Chromatogr. A 1172 (2007) 194-203, doi:10.1016/j.chroma.2007.10.006.

[17] R. Hecker, P.D. Fawell, A. Jefferson, J.B. Farrow, Flow field-flow fractionation of high-molecular-mass polyacrylamide, J. Chromatogr. A 837 (1999) 139-151, doi:10.1016/S0021-9673(99)00070-9.

[18] J.L. Viovy, Electrophoresis of DNA and other polyelectrolytes: Physical mechanisms. Rev. Mod. Phys. 72 (2000) 813-872, doi:10.1103/RevModPhys.72.813.

[19] J. P. Landers (Ed.), Handbook of Capillary Electrophoresis, CRC Press Inc.: Boca Raton, Florida, USA, 1996.

[20] N. Volpi (Ed.), Capillary Electrophoresis of Carbohydrates: From Monosaccharides to Complex Polysaccharides, Humana Press: New York, USA, 2011.

[21] V. Dolnik, Capillary gel electrophoresis, J. Microcol. Sep. $6 \quad$ (1994) 315-330, doi:10.1002/mcs.1220060402.

[22] H. Cottet, C. Simó, W. Vayaboury, A. Cifuentes, Nonaqueous and aqueous capillary electrophoresis of synthetic polymers, J. Chromatogr. A 1068 (2005) 59-73, doi:10.1016/j.chroma.2004.09.074.

[23] H. Cottet, P. Gareil, Capillary Electrophoresis: Methods and Protocols; P. Schmitt-Kopplin (Ed.), Humana Press: Totowa, NJ, USA, 2008, pp. 541-567.

[24] J. Chamieh, M. Martin, H. Cottet, Quantitative analysis in capillary electrophoresis: transformation of raw electropherograms into continuous distributions, Anal. Chem. 87 (2015) 1050-1057, doi:10.1021/ac503789s.

[25] J.J. Thevarajah, A. T. Sutton, A. R. Maniego, E. G. Whitty, S. Harrison, H. Cottet, P. Castignolles, M. Gaborieau, Quantifying the heterogeneity of chemical structures in complex charged polymers through the dispersity of their distributions of electrophoretic mobilities or of compositions, Anal. Chem. 88 (2016) 1674-1681, doi:10.1021/acs.analchem.5b03672.

[26] J.J. Thevarajah, M. Van Leeuwen, H. Cottet, P. Castignolles, M. Gaborieau, Determination of the distributions of degrees of acetylation of chitosan, Int. J. Biol. Macromol. 95 (2017) 40-48, doi:10.1016/j.ijbiomac.2016.10.056.

[27] H. Cottet, P. Gareil, O. Theodoly, C. Williams, A semi-empirical approach to the modeling of the electrophoretic mobility in free solution: Application to polystyrenesulfonates of various sulfonation rates, Electrophoresis 21 (2000) 3529-3540, doi:10.1002/1522-2683(200011)21:17\%3C3529::AIDELPS3529\%3E3.0.CO;2-2.

[28] G.S. Manning, Counterion binding in polyelectrolyte theory, Acc. Chem. Res. 12 (1979) 443-449, doi:10.1021/ar50144a004.

[29] D.A. Hoagland, D.L.Smisek, D.Y. Chen, Gel and free solution electrophoresis of variably charged polymers, Electrophoresis 17 (1996) 1151-1160, doi:10.1002/elps.1150170627.

[30] J.Y. Gao, P.L. Dubin, T. Sato, Y. Morishima, Separation of polyelectrolytes of variable compositions by free-zone capillary electrophoresis, J. Chromatogr. A 766 (1997) 233-236, doi:10.1016/S00219673(96)01037-0.

[31] H. Cottet, J.P. Biron, Charge- and size-based separations of polyelectrolytes by heart-cutting twodimensional capillary electrophoresis, Macromol. Chem. Phys. 206 (2005) 628-634, doi:10.1002/macp.200400538.

[32] I. Desvignes, J. Chamieh, H. Cottet, Separation and characterization of highly charged polyelectrolytes using free-solution capillary electrophoresis, Polymers 10 (2018) 1331, doi: 10.3390/polym10121331.

[33] C. Heller, Principles of DNA separation with capillary electrophoresis, Electrophoresis 22 (2001) 629643, doi:10.1002/1522-2683(200102)22:4<629::AID-ELPS629>3.0.CO;2-S.

[34] H. Cottet, P. Gareil, Electrophoretic behaviour of fully sulfonated polystyrenes in capillaries filled with entangled polymer solutuions, J. Chromatogr. A 772 (1997) 369-384, doi:10.1016/S00219673(96)01057-6.

[35] A. G. Ogston, The spaces in a uniform random suspension of fibres, Trans. Faraday Soc. 54 (1958) 1754-1757, doi :10.1039/TF9585401754.

[36] P.G. de Gennes. Scaling concept in polymer physics. Cornell University Press, Ithaca, NY, USA, 1979.

[37] G.W. Slater, J. Noolandi. On the reptation theory of gel electrophoresis, Biopolymers 25 (1986) 431454, doi:10.1002/bip.360250305.

[38] O.J. Lumpkin, P. Dejardin, B.H. Zimm. Theory of gel electrophoresis of DNA, Biopolymers 24 (1985) 1573-1593, doi:10.1002/bip.360240812. 
[39] P.D. Grossman, D.S. Soane, Experimental and theoretical studies of DNA separations by capillary electrophoresis in entangled polymer solutions, Biopolymers 31 (1991) 1221-1228, doi:10.1002/bip.360311010.

[40] H. Cottet, P. Gareil, J.L. Viovy, The effect of blob size and network dynamics on the size-based separation of polystyrenesulfonates by capillary electrophoresis in the presence of entangled polymer solutions, Electrophoresis 19 (1998) 2151-2162, doi:10.1002/elps.1150191219.

[41] E. Read, A. Guinaudeau, D.J. Wilson, A. Cadix, F. Violleau, M. Destarac, Low temperature RAFT/MADIX gel polymerisation: access to controlled ultra-high molar mass polyacrylamides, Polym. Chem. 5 (2014) 2202-2207, doi:10.1039/c3py01750h.

[42] A. Martínez-Richa, Determination of molecular size of O-(2-hydroxyethyl)cellulose (HEC) and its relationship to the mechanism of enzymatic hydrolysis by cellulases, Carbohydr. Polym. 87 (2012), 2129-2136, doi:10.1016/j.carbpol.2011.10.039.

[43] S. Magnúsdóttir, H. Isambert, C. Heller, J.L. Viovy, Electrohydrodynamically induced aggregation during constant and pulsed field capillary electrophoresis of DNA, Biopolymers 49 (1999) 385-401, doi:10.1002/(SICI)1097-0282(19990415)49:5<385::AID-BIP5>3.0.CO;2-L.

[44] B. Venzac, M. L. Diakité, D. Herthnek, I. Cissé, U. Bockelmann, S. Descroix, L. Malaquin, J.L. Viovy, On-chip conductometric detection of short DNA sequences via electro-hydrodynamic aggregation, Analyst 143 (2018) 190-199, doi:10.1039/C7AN00798A.

[45] M. Stefansson, Electrohydrodynamic instabilities and segregation of polysaccharides in capillary polymer solution electrophoresis, Biopolymers 49 (1999) 515-524, doi:10.1002/(SICI)10970282(199905)49:6<515::AID-BIP9>3.0.CO;2-E.

[46] A. Ibrahim, S. A. Allison, H. Cottet, Extracting information from the ionic strength dependence of the electrophoretic mobility by use of the slope-plot, Anal. Chem. 84 (2012) 9422-9430, doi:10.1021/ac302033z.

[47] H. Cottet, P. Gareil, On the use of the activation energy concept to investigate analyte and network deformation in entangled polymer solution capillary electrophoresis of synthetic polyelectrolytes, Electrophoresis 22 (2001) 684-691, doi:10.1002/1522-2683(200102)22:4<684::AIDELPS684>3.0.CO;2-O.

[48] A.E. Barron, W.M. Sunada, H.W. Blanch, Capillary electrophoresis of DNA in uncrosslinked polymer solutions: Evidence for a new mechanism of DNA separation, Biotechnol. Bioeng. 52 (1996) 259-270, doi:10.1002/(SICI)1097-0290(19961020)52:2<259::AID-BIT7>3.0.CO;2-P.

[49] Y. Vander Heyden, S.T. Popovici, B.B.P. Staal, P.J. Schoenmakers. Contribution of the polymer standards' polydispersity to the observed band broadening in size-exclusion chromatography, J. Chromatogr. A 986 (2003) 1-15, doi:10.1016/S0021-9673(02)01957-X.

[50] S.T. Popovici, W.T. Kok, P.J. Schoenmakers. Band broadening in size-exclusion chromatography of polydisperse samples, J. Chromatogr. A 1060 (2004), 237-252, doi:10.1016/j.chroma.2004.05.099. 
581 Figure 1. Free solution electropherograms of standards (APAM) obtained at $96.5 \mathrm{mM}$ ionic strength in time 582 scale (A) and in effective electrophoretic mobility scale (B). Experimental conditions: Fused silica capillary 40 $583 \mathrm{~cm}(30 \mathrm{~cm}$ to the detector $) \times 50 \mu \mathrm{m} \mathrm{ID}$. BGE: $20 \mathrm{mM}$ Tris/Cl buffer containing $85.5 \mathrm{mM} \mathrm{LiCl}, \mathrm{pH}$ 8. Separation 584 voltage: $+2 \mathrm{kV}$. Injection: $0.5 \mathrm{psi}, 3 \mathrm{~s}$. Sample concentration: $1 \mathrm{~g} / \mathrm{L}$ APAM $+0.05 \%$ DMF in BGE. UV detection

585

586

587

588

589

590

591

592

593

594

595

596

597

598

599

600

601

602

603

604

605

606

607

608

609

610

611 at $200 \mathrm{~nm}$. Temperature: $25^{\circ} \mathrm{C}$. Identification: DMF (1), APAM (2) and acylic acid (3). Acrylic acid was originally present in the polymer samples.

Figure 2. Electropherograms obtained for the size-based separation of APAM standards in HEC solution at $46.5 \mathrm{mM}$ ionic strength and $+2 \mathrm{kV}$ in time scale (A) and effective mobility scale (B). Experimental conditions: Fused silica capillary $40 \mathrm{~cm}(30 \mathrm{~cm}$ to the detector) $\times 50 \mu \mathrm{m}$ ID. BGE: HEC: $0.8 \mathrm{~g} / \mathrm{L}$ (blob size: $164 \mathrm{~nm})$ in 20 $\mathrm{mM}$ Tris/Cl buffer containing $35.5 \mathrm{mM} \mathrm{LiCl}, \mathrm{pH}$ 8. Electric voltage: $+2 \mathrm{kV}$. Injection: 0.5 psi, 3 s. Sample concentration: $1 \mathrm{~g} / \mathrm{L}$ APAM + $0.05 \%$ DMF. UV detection at $200 \mathrm{~nm}$. Temperature: $25^{\circ} \mathrm{C}$. Identification: DMF (1), APAM (2), acrylic acid (3).

Figure 3. Electropherograms obtained for the size-based separation of APAM standards in HEC solution at 96.5 $\mathrm{mM}$ ionic strength in time scale (A, C) and effective mobility scale (B, D) at different injected concentrations (A, B: $2 \mathrm{~g} / \mathrm{L}$; and C, D: $1.5 \mathrm{~g} / \mathrm{L})$. Experimental conditions: Fused silica capillary $40 \mathrm{~cm}(30 \mathrm{~cm}$ to the detector $) \times 50$ $\mu \mathrm{m}$ ID. BGE: HEC: $0.8 \mathrm{~g} / \mathrm{L}$ (blob size $164 \mathrm{~nm}$ ) in $20 \mathrm{mM}$ Tris/Cl buffer containing $85.5 \mathrm{mM} \mathrm{LiCl,} \mathrm{pH} 8$. Electric voltage: $+2 \mathrm{kV}(50 \mathrm{~V} / \mathrm{cm})$. Injection: $0.5 \mathrm{psi}, 3 \mathrm{~s}$. Sample concentration: $1.5 \mathrm{~g} / \mathrm{L}(\mathrm{C}, \mathrm{D})$ or $2 \mathrm{~g} / \mathrm{L}(\mathrm{A}, \mathrm{B})$ $\mathrm{APAM}+0.05 \% \mathrm{DMF}$. UV detection at $200 \mathrm{~nm}$. Temperature: $25^{\circ} \mathrm{C}$. Identification: DMF (1), APAM (2), mobility marker: anthraquinone sulfonic acid (3).

Figure 4. Reduced electrophoretic mobility distributions $\mathrm{P}\left(\mu / \bar{\mu}_{0}\right)$ of APAM satandards (A) and IPAM industrial samples (B) in $0.8 \mathrm{~g} / \mathrm{L}$ HEC Tris/Cl electrolyte at $96 \mathrm{mM}$ ionic strength. Experimental conditions as in Fig. 3. Injected concentrations at $1.5 \mathrm{~g} / \mathrm{L}(\mathrm{A})$ and $1 \mathrm{~g} / \mathrm{L}(\mathrm{B})$.

Figure 5. $\log \left(\bar{\mu} / \bar{\mu}_{0}\right)$ vs $\log M_{w}$ plot of APAM standard $(\bullet)$ in $0.8 \mathrm{~g} / \mathrm{L}$ HEC solution and for IPAM industrial samples with $M_{w}$ from SLS $(\boldsymbol{\nabla})$. The calibration curve obtained on the APAM samples reads: $\log \left(\bar{\mu} / \bar{\mu}_{0}\right)=-$ $(0.1129 \pm 0.0069) \log M w-(0.109 \pm 0.0040)\left(M_{w}\right.$ in $\left.\mathrm{g} / \mathrm{mol}\right), R^{2}=0.9864$. Expected molar masses for IPAM industrial samples $(\bullet)$ obtained by CGE by integration of the polymer peak of Fig. $6 \mathrm{~B}$ using equation (8). Experimental conditions as in Fig. 3.

Figure 6. Area-normalized molar mass distribution $(\mathrm{P}(\log M) \sim \mathrm{P}(M) \times M)$ of APAM standards $(\mathrm{A})$ and IPAM industrial samples (B) in HEC solution. Experimental conditions as in Fig. 3. 